\title{
Pressure Sore
}

National Cancer Institute

\section{Source}

National Cancer Institute. Pressure Sore. NCI Thesaurus. Code C50706.

Death of tissue due to external pressure. 